\title{
Interventional Stroke Treatment: Challenges after MR CLEAN
}

\author{
M. Bendszus
}

Published online: 14 February 2015

(C) Springer-Verlag Berlin Heidelberg 2015

Many neuro-interventionalists anxiously expected the results of the MR CLEAN trial, which was feared to be negative because of relatively broad inclusion criteria and low requirements for the interventionalists. Fortunately, all primary and secondary analyses were in favor of endovascular stroke treatment. After the interim analysis of ESCAPE and EXTEND-IA, which reported to be positive as well, most of ongoing stroke trials were halted and analyzed prematurely. Is this a won war or just a victorious battle? By these data, an important if not essential step has been made to establish endovascular stroke treatment once for all.

However, important further steps need to be made: most importantly, data of MR CLEAN need to be confirmed by additional randomized control trials to gain a level of evidence that warrants nationwide coverage of endovascular stroke treatment. Furthermore, all present endovascular stroke trials have been looking at the "bright side" of stroke treatment, i.e., treating patients with a relatively good prognosis and selecting a good outcome as primary endpoint. In future trials, we also have to address the "dark side" of stroke treatment, i.e., patients with a worse prognosis (due to later time window or more extensive early ischemia) where we hope to reduce (but not prevent) bad outcome, rates of craniectomy and mortality. Finally, we as neuroradiological community have to be ready for prime time, which is the time when endovascular stroke treatment is the nationwide standard in severe acute stroke. To guarantee a comprehensive service, we have to dramatically increase our training efforts in interventional neuroradiology, not necessarily to educate neuroradiologists treating all vascular lesions from stroke to AVMs but also "lower level" capable of treating acute ischemic stroke. If we do not manage to cover this need, other specialties will be more than pleased to help.

M. Bendszus $(\square)$

Department of Neuroradiology, University of Heidelberg,

Heidelberg, Germany

e-mail: Martin.Bendszus@med.uni-heidelberg.de 